\title{
PSEUDOCOMPACT TOTALLY DENSE SUBGROUPS
}

\author{
DIKRAN DIKRANJAN AND ANNA GIORDANO BRUNO
}

(Communicated by Alexander N. Dranishnikov)

\begin{abstract}
It was shown by Dikranjan and Shakhmatov in 1992 that if a compact abelian group $K$ admits a proper totally dense pseudocompact subgroup, then $K$ cannot have a torsion closed $G_{\delta}$-subgroup; moreover this condition was shown to be also sufficient under LH. We prove in ZFC that this condition actually ensures the existence of a proper totally dense subgroup $H$ of $K$ that contains an $\omega$-bounded dense subgroup of $K$ (such an $H$ is necessarily pseudocompact). This answers two questions posed by Dikranjan and Shakhmatov (Proc. Amer. Math. Soc. 114 (1992), 1119-1129).
\end{abstract}

\section{INTRODUCTION}

A subgroup $H$ of a topological abelian group $G$ is said to be totally dense if $H$ densely intersects every closed subgroup of $G$ [15]. The interest in the totally dense subgroups of a compact abelian group $K$ stems from the fact that they are precisely those dense subgroups of $K$ that satisfy the open mapping theorem $[13,7,8]$. In other words the totally dense subgroups of a compact abelian group $K$ have a compactness-like property. Therefore it is natural to expect that in the presence of other compactness-like properties they may turn out to be actually compact, hence coincide with $K$. Indeed no compact abelian group $K$ has a proper totally dense countably compact subgroup [9, Theorem 1.4]. We recall here that a topological group $G$ is countably compact if every countable open cover of $G$ has a finite subcover, $G$ is pseudocompact if every continuous real-valued function of $G$ is bounded, and $G$ is $\omega$-bounded if every countable subset of $G$ is contained in a compact subgroup of $G$. The following chain of implications holds:

$$
\text { compact } \Rightarrow \omega \text {-bounded } \Rightarrow \text { countably compact } \Rightarrow \text { pseudocompact. }
$$

Comfort and Soundararajan [4] first studied the question of when a compact abelian group $K$ admits a proper totally dense pseudocompact subgroup and provided a solution in the case when $K$ is connected (iff $K$ is not metrizable). Later Comfort and Robertson [2] studied the compact abelian groups $K$ that admit a totally dense pseudocompact subgroup of size $<|K|$. They showed that $Z F C$ cannot decide whether there exists a compact abelian group $K$ with this property $[2$, Theorem 6.3].

For the sake of brevity let us give the following definition (see [5, Definition 1.2]).

Received by the editors May 8, 2006 and, in revised form, October 23, 2006.

2000 Mathematics Subject Classification. Primary 22A05, 54H11; Secondary 22C05, 54D25.

This work was partially supported by a PRIN2005 grant of the Italian MIUR and by funds of the PhD program at the Department of Mathematics of the University of Udine. 
Definition 1.1. A compact abelian group $K$ has the property $T D_{\omega}$ if there exists a proper totally dense subgroup $H$ of $K$ that contains an $\omega$-bounded dense subgroup.

Since the subgroup $H$ must be pseudocompact, the property $T D_{\omega}$ implies that $K$ has a proper totally dense pseudocompact subgroup. In these terms the following theorem was proved, extending the ideas from [4] (a self-contained proof of this theorem is given in $\S 2$ for the reader's convenience).

Theorem 1.2 ([9, Theorem 1.9]). Every compact abelian group with non-metrizable connected component of zero has the property $T D_{\omega}$.

The following necessary condition for the existence of proper totally dense pseudocompact subgroups of compact abelian groups was given in [9, Theorem 1.7].

Theorem 1.3. Let $G$ be an abelian topological group which has a torsion, closed $G_{\delta}$-subgroup $N$. Then $G$ contains no proper totally dense pseudocompact subgroups.

Moreover, under the additional set-theoretic assumption $2^{\omega_{1}}=\mathfrak{c}$, known under the name of Lusin's Hypothesis (LH), this necessary condition (obviously implying non-metrizablity) was shown to be the unique constraint for the existence of proper totally dense pseudocompact subgroups of compact abelian groups:

Theorem 1.4 ([9, Theorem 1.8]). Under LH a compact abelian group $K$ contains a proper totally dense pseudocompact subgroup if and only if $K$ has no torsion, closed $G_{\delta}$-subgroups.

One of the implications of Theorem 1.4 is Theorem 1.3. To prove the other one in [9] the authors first proved it in case the group $K$ has cardinality $\mathfrak{c}$. In particular, under the assumption of LH, the implication holds true for groups $K$ of weight $\omega_{1}$ (as $|K|=2^{\omega_{1}}=\mathfrak{c}$ ). In the general case, supposing that $K$ has no torsion, closed $G_{\delta}$-subgroups, they constructed a continuous surjective homomorphism from $K$ to a compact abelian group $H$ of weight $\omega_{1}$ with the same property. Under LH $H$ has a proper totally dense pseudocompact subgroup. The inverse image of this subgroup of $H$ is a proper totally dense pseudocompact subgroup of $K$.

One of the open problems left in [9, Problem 1.11] was whether the assumption of LH in Theorem 1.4 can be removed. Another open problem left there [9, Problem 1.12] was whether a compact abelian group without torsion, closed $G_{\delta}$-subgroups has the property $T D_{\omega}$. The main result of our paper (exposed in the next theorem) resolves positively both problems providing, as a by-product, also independent and shorter proofs of Theorems 1.2 and 1.4 as well as of the conjecture made in [5, Remark 3.20].

Theorem 1.5. For a compact abelian group $K$ the following conditions are equivalent:

(a) $K$ has a proper totally dense pseudocompact subgroup;

(b) $K$ has no closed, torsion $G_{\delta}$-subgroups;

(c) $K$ has the property $T D_{\omega}$.

The proof of Theorem 1.5 is quite different from that of Theorem 1.4 given in [9]. In fact, following [5] we add a third condition, that is, $K$ has the property $T D_{\omega}$, which seems to be stronger than the others, but it turns out to be equivalent to them and it helps to prove the theorem in its full generality.

The plan of the proof is the following. It is easy to see that all these conditions imply the non-metrizability of $K$. The groups $K$ with non-metrizable connected 
component $c(K)$ are settled by Theorem 1.2. In case $c(K)$ is metrizable one can consider the totally disconnected quotient $K_{1}=K / c(K)$ that is again non-metrizable, provided $K$ is non-metrizable. Now every proper totally dense pseudocompact subgroup $H$ of $K_{1}$ defines (via inverse image) a proper totally dense pseudocompact subgroup of $K$ (see Lemma 2.5). In this way the main difficulty is confined in the totally disconnected case. In $\S 4$ we provide various results on the structure of totally disconnected compact abelian groups; this allows us to resolve the problem in the "local case" (i.e., for abelian pro- $p$-groups) in Lemma 5.1. Then this is applied in the proof of the general case given in $\S 5$.

Example 1.6. Assuming LH, there exists a compact abelian group that has a totally dense pseudocompact subgroup which does not contain any non-trivial dense $\omega$-bounded subgroup (we give the construction of such an example in $\S 5$ ).

In a forthcoming paper [12], the second-named author characterizes (in a way analogous to Theorem 1.5) the compact abelian groups $K$ which admit a proper dense pseudocompact subgroup $H$ such that $H$ non-trivially intersects each nontrivial closed subgroup of $K$ (obviously totally dense subgroups have this property).

Notation and terminology. We denote by $\mathbb{Z}, \mathbb{P}, \mathbb{N}$, and $\mathbb{N}_{+}$respectively the set of integers, the set of primes, the set of natural numbers, and the set of positive integers. For $m \in \mathbb{N}_{+}$, we use $\mathbb{Z}(m)$ for the finite cyclic group of order $m$. The circle group $\mathbb{T}$ is identified with the quotient group $\mathbb{R} / \mathbb{Z}$ of the reals $\mathbb{R}$ and carries its usual topology. For a prime $p \in \mathbb{P}$ the symbol $\mathbb{Z}_{p}$ is used for the group of $p$-adic integers.

Let $G$ be an abelian group. The subgroup of torsion elements of $G$ is $t(G)$ and $G[m]$ is the subset of all elements $x$ of $G$ such that $m x=0$. We denote by $r_{0}(G)$ the free-rank of $G$. The symbol $\mathfrak{c}$ stands for the cardinality of the continuum.

Throughout this paper all topological groups are Hausdorff. For a topological group $G$ we use $w(G)$ to indicate the weight of $G$, that is, the minimal cardinality of a base for the topology of $G$. We denote by $c(G)$ the connected component of 0 in $G$. If $c(G)$ is trivial, the group $G$ is said to be totally disconnected. If $M$ is a subset of $G$, then $\langle M\rangle$ is the smallest subgroup of $G$ containing $M$ and $\bar{M}$ is the closure of $M$ in $G$. The Pontryagin dual of a topological abelian group $G$ is denoted by $\widehat{G}$. For a product $K^{\sigma}$ of a group $K$ with $\sigma>\omega$ we denote by $\Sigma K^{\sigma}$ the $\Sigma$-product centered at 0 of $K^{\sigma}$, that is, the set of all elements with support of countable cardinality. For undefined terms see [10].

\section{Groups With the Property $T D_{\omega}$}

Let us give an example of a group that has the property $T D_{\omega}$.

Example 2.1 ([5, Example 3.15]). Let $K_{p}=\mathbb{Z}(p)^{\omega_{1}}$ and $K=\prod_{p \in \mathbb{P}} K_{p}$. Note that $K=S^{\omega_{1}}$ with $S=\prod_{p \in \mathbb{P}} \mathbb{Z}(p)$. The subgroup $H=\Sigma S^{\omega_{1}}+t(K)$ is a proper totally dense pseudocompact subgroup of $K$ since $t(K)=\bigoplus_{p \in \mathbb{P}} K_{p}$ is totally dense in $K$, while $\Sigma S^{\omega_{1}}$ is a dense $\omega$-bounded (hence pseudocompact) subgroup of $K$.

In this example the idea from [2] is used to construct the totally dense pseudocompact subgroups as sums of two subgroups: one totally dense and the other dense pseudocompact. The problem in general is to ensure that such a sum is a proper subgroup. Lemma 2.3 offers a completely different solution. First, we need the following lemma. 
Lemma 2.2. Let $K$ be an abelian group, $C$ a torsion-free subgroup of $K$, and $B$ a subgroup of $K$ maximal with the property $B \cap C=\{0\}$. Then $B \cap m N=m(B \cap N)$ for every $0 \neq m \in \mathbb{Z}$ and for every subgroup $N$ of $K$. In particular, if $p \in \mathbb{P}$, then $B \cap N \nsubseteq p N$ for every subgroup $N$ of $K$ isomorphic to $\mathbb{Z}_{p}$, whenever $r_{0}(C)<\mathfrak{c}$.

Proof. If $x \in B \cap m N$, then $x=m z$, with $z \in N$. Assume that $z \notin B$. It follows that $B$ is a proper subgroup of $B_{1}=B+\langle z\rangle$. So there exists $y \in B_{1} \cap C, y \neq 0$, that is, $y=b+k z \in C$, where $b \in B$ and $k \in \mathbb{Z}$. Then $m y=m b+k m z \in B \cap C=\{0\}$ and hence $m y=0$. As $C$ is torsion free, we conclude that $y=0$, finding a contradiction.

Suppose that $r_{0}(C)<\mathfrak{c}$ and assume for a contradiction that $N$ is a subgroup of $K$ isomorphic to $\mathbb{Z}_{p}$ with $B \cap N \subseteq p N$. Then $B \cap N=B \cap p N$. Applying the first part we have also $B \cap p N=p(B \cap N)=p(B \cap p N)$. By induction $B \cap p N=p^{n}(B \cap p N)$ for every $n \in \mathbb{N}$. Hence $B \cap p N=\bigcap_{n=1}^{\infty} p^{n}(B \cap p N)$, but $\bigcap_{n=1}^{\infty} p^{n}(B \cap N) \subseteq \bigcap_{n=1}^{\infty} p^{n} N=\{0\}$. So $B \cap N=\{0\}$. If $\varphi$ is the natural projection of $K$ onto $K / B$, this yields that $\varphi \uparrow_{N}$ is injective and consequently $r_{0}(K / B) \geq r_{0}(N)=\mathfrak{c}$.

To get a contradiction we prove that $r_{0}(K / B) \leq r_{0}(C)<\mathfrak{c}$. Suppose that $r_{0}(K / B)>r_{0}(C)$. Then $\varphi(C)$ is a torsion-free subgroup of $K / B$ such that $r_{0}((K / B) / \varphi(C)) \geq 1$. So there exists an infinite cyclic subgroup $C_{1}$ of $K / B$ such that $C_{1} \cap \varphi(C)=\{0\}$. Now take $\varphi^{-1}\left(C_{1}\right)$ and observe that $\varphi^{-1}\left(C_{1}\right) \cap C=B \cap C=$ $\{0\}$ and $\varphi^{-1}\left(C_{1}\right) \supsetneq \operatorname{ker} \varphi=B$, contradicting the maximality of $B$.

Now we are able to prove the following lemma, that produces totally dense subgroups containing a given subgroup. It was announced without a proof in $[5$, Lemma 3.16].

Lemma 2.3. Let $K$ be a compact abelian group that admits a subgroup $B$ such that $r_{0}(K / B) \geq 1$. Then $K$ has a proper totally dense subgroup $H$ that contains $B$.

Proof. Since $r_{0}(K / B) \geq 1$, there exists a cyclic infinite subgroup $C$ of $K$ such that $B \cap C=\{0\}$. Let $B_{1}=B+t(K)$; then $B_{1} \cap C=\{0\}$. By Zorn's Lemma there exists a subgroup $H$ of $K$ such that $H \supseteq B_{1}, H \cap C=\{0\}$ and $H$ is maximal with respect to these two properties. It immediately follows that $H \supseteq B$ and that $H$ is a proper subgroup of $K$. Moreover $t(K) \subseteq H$ and by Lemma $2.2 H \cap N \nsubseteq p N$ holds for every subgroup $N$ of $K$ isomorphic to $\mathbb{Z}_{p}$ and for every $p \in \mathbb{P}$. Now apply the local criterion for total density [8, Theorem 4.3.7] to conclude that $H$ is totally dense in $K$.

A subgroup $H$ of a topological group $G$ is $G_{\delta}$-dense in $G$ if it non-trivially intersects each $G_{\delta}$-subset of $G$. If $K$ is a compact group, by the Comfort and Ross theorem [3] a dense subgroup $H$ of $K$ is pseudocompact if and only if it is $G_{\delta}$-dense in $K$. Therefore, if the subgroup $B$ of $K$ in Lemma 2.3 is dense and pseudocompact, then $H$ is pseudocompact too. This will be used when this lemma will be applied in the following proposition, that in particular covers Example 2.1.

Proposition 2.4 ([5, Example 3.17]). Let $K$ be a non-torsion compact abelian group. Then $K^{\omega_{1}}$ has the property $T D_{\omega}$. In particular $\mathbb{T}^{\omega_{1}}$ has the property $T D_{\omega}$.

Proof. In $K^{\omega_{1}}$ the $\Sigma$-product $\Sigma K^{\omega_{1}}$ is a dense $\omega$-bounded subgroup which meets trivially the diagonal subgroup $\Delta_{K} \omega_{1}$. Since $\Delta_{K}{ }^{\omega_{1}} \cong K$, consequently $r_{0}\left(\Delta_{K^{\omega_{1}}}\right) \geq$ $\mathfrak{c}$, that is, $r_{0}\left(K^{\omega_{1}} / \Sigma K^{\omega_{1}}\right) \geq \mathfrak{c}$. Now apply Lemma 2.3 to conclude that $K^{\omega_{1}}$ has the property $T D_{\omega}$. 
Here we give a basic property of condition $T D_{\omega}$, that will be used in the proof of our Main Theorem 5.2.

Lemma 2.5 ([5, Lemma 3.12]). Let $K$ be a compact abelian group that admits a continuous surjective homomorphism $f$ onto a compact abelian group $L$. If $L$ has the property $T D_{\omega}$, then $K$ has the property $T D_{\omega}$ too.

Proof. There exists a proper totally dense subgroup $H$ of $L$ such that $H$ contains an $\omega$-bounded dense subgroup $M$. So $f^{-1}(H)$ is a proper totally dense subgroup of $K$ and it contains $f^{-1}(M)$ which is dense and $\omega$-bounded.

Proof of Theorem 1.2. A standard application of Pontryagin duality shows that $K$ admits a surjective continuous homomorphism onto $\mathbb{T}^{\omega_{1}}$. Now Lemma 2.5 can be applied, as $\mathbb{T}^{\omega_{1}}$ has the property $T D_{\omega}$ by Proposition 2.4 .

\section{Singular Groups}

In order to find a more "algebraic" form of the condition of Theorem 1.3 and Theorem 1.4, we note that for a compact abelian group $K$ the family $\Lambda(K)$ of all closed $G_{\delta}$-subgroups of $K$ is closed under countable intersections. Moreover $N \in \Lambda(K)$ if and only if $K / N$ is metrizable.

Definition 3.1. A compact abelian group $K$ is singular if there exists $m \in \mathbb{N}_{+}$ such that $K[m] \in \Lambda(K)$.

The next lemma offers an alternative form for singularity of compact abelian groups ( $m K$ is metrizable compact for some $m \in \mathbb{N}_{+}$). It is useful while checking stability of this property under taking subgroups and quotients.

Lemma 3.2 ([6, Lemma 4.1]). Let $G$ be a topological abelian group and $m \in \mathbb{N}_{+}$.

(a) If $m G$ is metrizable, then $G[m] \in \Lambda(G)$.

(b) If $G$ is compact, then $G[m] \in \Lambda(G)$ implies that $m G$ is metrizable.

The following lemma gives a condition equivalent to the hypothesis of Theorem 1.3 in terms of singular groups.

Lemma 3.3. Let $K$ be a compact abelian group. Then $K$ is singular if and only if there exists a torsion subgroup $N \in \Lambda(K)$.

Proof. Obviously, if $K$ is singular, $K[n]$ is torsion and $K[n] \in \Lambda(K)$ for some $n \in \mathbb{N}_{+}$. If $N \in \Lambda(K)$ is torsion, then by the compactness of $N$ there exists $n \in \mathbb{N}_{+}$such that $n N=\{0\}$. Therefore $N$ is contained in the subgroup $K[n]$ of $K$. Consequently $K[n] \in \Lambda(K)$.

Lemma 3.4. Let $K$ be a compact abelian group and let $N$ be a closed subgroup of $K$. Then $K$ is singular if and only if $N$ and $K / N$ are singular. In particular finite products of singular compact abelian groups are singular.

Proof. The necessity is obvious. Let us prove the sufficiency. Suppose that both $N$ and $K / N$ are singular and let $X$ and $Y$ be the Pontryagin duals of $K$ and $K / N$ respectively. We say that $X$ is almost-countable if $m X$ is countable for some $m \in \mathbb{N}_{+}$. By Pontryagin duality $K$ is singular if and only if $X$ is almost-countable. 
Since $Y$ can be identified with a subgroup of $X$ such that $X / Y \cong \widehat{N}$, both $Y$ and $X / Y$ are almost-countable. Thus there exists $m \in \mathbb{N}_{+}$such that $m Y$ and $m(X / Y)$ are countable. Since $m(X / Y)=(m X+Y) / Y$ is countable, $m X$ is contained in a countable union $\bigcup_{n=1}^{\infty}\left(z_{i}+Y\right)$, where $z_{i} \in m X$. As $m Y$ is countable, $m^{2} X$ is contained in the union $\bigcup_{n=1}^{\infty}\left(m z_{i}+m Y\right)$, which is countable. So $X$ is almostcountable.

\section{Non-SINGULAR PRO- $p$-GROUPS}

For an abelian topological group $G$ and a prime $p$ we denote by $G_{p}$ the topological $p$-component of $G$, that is, $G_{p}=\left\{x \in G: p^{n} x \rightarrow 0\right.$ for some $\left.n \in \mathbb{N}\right\}$. By [1] a totally disconnected non-metrizable compact abelian group $K$ can be decomposed as $K=\prod_{p} K_{p}$. Each $K_{p}$ is an abelian pro- $p$-group (i.e., projective limit of finite $p$-groups) and at least one of the $K_{p}$ is non-metrizable.

As we said above, the main difficulty of the problem is in the totally disconnected case. So in this section we consider the case when $K$ is a totally disconnected compact abelian group in relation to singularity and metrizability of the topological $p$-components of $K$. In particular Example 2.1 shows that it may happen that each $K_{p}$ is singular even if the product $K$ is non-singular.

Lemma 4.1. Let $p$ be a prime and let $K$ be an abelian pro-p-group.

(a) If $K / p K$ is finite, then $K \cong \mathbb{Z}_{p}^{m} \times F$, where $m \in \mathbb{N}$ and $F$ is a finite p-group.

(b) If $K / p K$ is infinite, then $w(K)=w(K / p K)$.

In particular $K$ is metrizable whenever $K / p K$ is metrizable.

Proof. By Pontryagin duality $X=\widehat{K}$ is a $p$-group and $X[p]$ is the dual of the quotient $K / p K$.

(a) Assume that $K / p K$ is finite. Then $X[p]$ is finite; hence $X[p]$ is isomorphic to a subgroup of $\mathbb{Z}\left(p^{\infty}\right)^{n}$, where $n$ is the $p$-rank of $X$ (that is, the dimension of $X[p]$ as a vector space over the field $\mathbb{Z} / p \mathbb{Z})$. Since $\mathbb{Z}\left(p^{\infty}\right)^{n}$ is divisible, this immersion can be extended to $j: X \rightarrow \mathbb{Z}\left(p^{\infty}\right)^{n}$. Now $j$ is injective because if $x \in$ ker $j$ and $x \neq 0$, then we can suppose wlog that $p x=0$, that is, $x \in X[p]$ and this is not possible. If $d(X)$ is the maximal divisible subgroup of $X$, then $d(X)$ is isomorphic to $\mathbb{Z}\left(p^{\infty}\right)^{m}$ with $m \leq n$. Thus $X \cong \mathbb{Z}\left(p^{\infty}\right)^{m} \oplus X_{1}$ where $X_{1}$ is reduced and so finite because it has finite $p$-rank [11]. By Pontryagin duality $K=\widehat{X} \cong \mathbb{Z}_{p}^{m} \times F$, where $F=\widehat{X_{1}} \cong X_{1}$ is finite.

(b) follows from the fact that $|X|=|X[p]|$, when $X[p]$ is infinite.

Remark 4.2. Let $K$ be a totally disconnected compact abelian group. So $K=$ $\prod_{p \in \mathbb{P}} K_{p}$. Define

$$
P_{s}=\left\{p \in \mathbb{P}: K_{p} \text { is singular }\right\} \text { and } P_{m}=\left\{p \in \mathbb{P}: K_{p} \text { is metrizable }\right\},
$$

and observe that $P_{m} \subseteq P_{s} \subseteq \mathbb{P}$. The group $K$ is metrizable if and only if $P_{m}=\mathbb{P}$.

Suppose that $K$ is non-metrizable. Let $K_{m}=\prod_{p \in P_{m}} K_{p}, K_{s}=\prod_{p \in P_{s} \backslash P_{m}} K_{p}$, and $K_{r}=\prod_{p \in \mathbb{P} \backslash P_{s}} K_{p}$. If $P_{m}$ (resp. $P_{s} \backslash P_{m}, \mathbb{P} \backslash P_{m}$ ) is empty, put $K_{m}=\{0\}$ (resp. $\left.K_{s}=\{0\}, K_{r}=\{0\}\right)$. Note that $K=K_{m} \times K_{s} \times K_{r}$ and that $K_{m}$ is metrizable.

The next proposition takes care of the case when $\mathbb{P} \backslash P_{m}$ is infinite. 
Proposition 4.3. Let $K$ be a totally disconnected compact abelian group such that $\mathbb{P} \backslash P_{m}$ is infinite. Then there exists a continuous surjective homomorphism of $K$ onto $S^{\omega_{1}}$, where $S$ is a non-torsion metrizable compact abelian group.

Proof. By Lemma 4.1 there exist infinitely many primes $p_{n}\left(n \in \mathbb{N}_{+}\right)$such that $K / p_{n} K$ is non-metrizable. Since $K / p_{n} K$ is a non-metrizable compact abelian group of exponent $p_{n}$, it is topologically isomorphic to $\mathbb{Z}\left(p_{n}\right)^{w\left(K / p_{n} K\right)}$, where $w\left(K / p_{n} K\right) \geq$ $\omega_{1}$. This yields that for every $n \in \mathbb{N}_{+}$there exists a continuous surjective homomorphism $f_{n}: K / p_{n} K \rightarrow \mathbb{Z}\left(p_{n}\right)^{\omega_{1}}$. As $K_{q}=p_{n} K_{q} \subseteq p_{n} K$ for every prime $q \neq p_{n}$, one gets $p_{n} K=p_{n} K_{p_{n}} \times \prod_{q \neq p_{n}} K_{q}$. So $K / p_{n} K \cong K_{p_{n}} / p_{n} K_{p_{n}}$ and consequently $f_{n}$ can be identified with $f_{n}^{\prime}: K_{p_{n}} / p_{n} K_{p_{n}} \rightarrow \mathbb{Z}\left(p_{n}\right)^{\omega_{1}}$. With $S=\prod_{n=1}^{\infty} \mathbb{Z}\left(p_{n}\right)$ this gives a continuous surjective homomorphism

$$
f=\prod_{n=1}^{\infty} f_{n}^{\prime}: \prod_{n=1}^{\infty} K_{p_{n}} / p_{n} K \rightarrow \prod_{n=1}^{\infty} \mathbb{Z}\left(p_{n}\right)^{\omega_{1}}=S^{\omega_{1}} .
$$

Hence the composition of the continuous surjective homomorphism $K=\prod_{p \in \mathbb{P}} K_{p} \rightarrow$ $\prod_{n=1}^{\infty} K_{p_{n}} / p_{n} K_{p_{n}}$ with $f$ is a continuous surjective homomorphism of $K$ onto $S^{\omega_{1}}$.

Lemma 4.4. Let $K$ be a totally disconnected compact abelian group. Then $K$ is singular if and only if $P_{s}=\mathbb{P}$ and $\mathbb{P} \backslash P_{m}$ is finite.

Proof. Suppose that $P_{s}=\mathbb{P}$ and $\mathbb{P} \backslash P_{m}$ is finite. Then $K=K_{m} \times K_{s}$, where $K_{m}$ is metrizable and $K_{s}$ is singular by Lemma 3.4. So $K$ is singular.

Now we prove the converse implication. If $\mathbb{P} \backslash P_{m}$ is infinite, by Proposition 4.3 there exists a continuous surjective homomorphism of $K$ onto $S^{\omega_{1}}$, where $S$ is a non-torsion metrizable compact abelian group. Since $S^{\omega_{1}}$ is non-singular, then $K$ is non-singular by Lemma 3.4. If $\mathbb{P} \neq P_{s}$ then Lemma 3.4 implies that $K$ is non-singular.

Lemma 4.5. Let $\sigma$ be a cardinal, $K$ an abelian pro-p-group, and $N$ a closed subgroup of $K$ isomorphic to $\mathbb{Z}_{p}^{\sigma}$ such that $K / N$ is singular. Then $K$ is non-singular if and only if $\sigma>\omega$.

Proof. If $\sigma>\omega$, then $N$ is non-singular and so again Lemma 3.4 implies that $K$ is non-singular too. Suppose that $\sigma \leq \omega$. Then $N$ is metrizable and in particular $N$ is singular. By Lemma 3.4 this implies that $K$ is singular.

In the sequel we denote by $\mathbb{L}_{p}$ the group $\prod_{n=1}^{\infty} \mathbb{Z}\left(p^{n}\right)$.

Proposition 4.6. Let $K$ be an abelian pro-p-group. Then $K$ has a closed subgroup $N$ such that

(a) $N \cong \mathbb{Z}_{p}^{\sigma}$ for some cardinal $\sigma$;

(b) the quotient $L=K / N$ is isomorphic to $\prod_{n=1}^{\infty} \mathbb{Z}\left(p^{n}\right)^{\alpha_{n}}$, for some cardinals $\alpha_{n}$

(c) if $\varphi$ is the canonical projection of $K$ onto $L$, then $\varphi(t(K))=t(L)$.

Moreover, if $K$ is non-singular, then:

(1) there exists a continuous surjective homomorphism of $K$ onto $\mathbb{L}_{p}^{\omega_{1}}$, in case $L$ is non-singular;

(2) $\sigma=w(N)>\omega$, in case $L$ is singular. 
Proof. Let $X=\widehat{K}$. For a subset $T$ of $K$ the annihilator of $T$ in $X$ is $T^{\perp}=\{\chi \in$ $X: \chi(T)=\{0\}\}$.

(a), (b) By [11, Theorem 32.3] $X$ contains a pure subgroup $B$ (i.e., $p^{n} X \cap B=$ $p^{n} B$ for every $\left.n \in \mathbb{N}\right)$ such that $B \cong \bigoplus_{n=1}^{\infty} \mathbb{Z}\left(p^{n}\right)^{\left(\alpha_{n}\right)}$ and $X / B \cong \mathbb{Z}\left(p^{\infty}\right)^{(\sigma)}$ for some cardinals $\sigma, \alpha_{n}$. By Pontryagin duality $K$ has a closed subgroup $N \cong \mathbb{Z}_{p}^{\sigma}$ (namely the annihilator of $B$ ) such that the quotient $L=K / N$ is isomorphic to $\prod_{n=1}^{\infty} \mathbb{Z}\left(p^{n}\right)^{\alpha_{n}}$.

(c) To prove that $\varphi(t(K))=t(L)$ we show that $\varphi\left(K\left[p^{n}\right]\right)=L\left[p^{n}\right]$ holds for every $n \in \mathbb{N}_{+}$. It is sufficient to see that $W=\left\{x \in K: p^{n} x \in N\right\}=N+K\left[p^{n}\right]$. Since $W=\varphi^{-1}\left(L\left[p^{n}\right]\right)$, obviously $W$ contains $N+K\left[p^{n}\right]$. Since $W$ and $N+K\left[p^{n}\right]$ are closed subgroups, to prove that they coincide it suffices to prove that their annihilators coincide. From $W \supseteq N+K\left[p^{n}\right]$, it follows $W^{\perp} \subseteq N^{\perp} \cap K\left[p^{n}\right]^{\perp}=$ $B \cap p^{n} X=p^{n} B$. Observe that the last equality follows from the purity of $B$. But we have also

$$
W^{\perp}=\left\{\chi \in X:(\forall x \in K) p^{n} x \in N \Rightarrow \chi(x)=0\right\} \supseteq p^{n}\left(N^{\perp}\right)=p^{n} B .
$$

Therefore $W^{\perp}=N^{\perp} \cap K\left[p^{n}\right]^{\perp}$.

Suppose now that $K$ is non-singular.

(1) If $L$ is non-singular, $p^{n} L$ is non-metrizable for every $n \in \mathbb{N}_{+}$. Since $p^{n} L \cong$ $\prod_{m=n+1}^{\infty} \mathbb{Z}\left(p^{m-n}\right)^{\alpha_{m}}$, there are infinitely many $\alpha_{n}>\omega$. Hence there exists a continuous surjective homomorphism of $L$ onto $\prod_{n=1}^{\infty} \mathbb{Z}\left(p^{n}\right)^{\omega_{1}} \cong \mathbb{L}_{p}^{\omega_{1}}$. Combining it with $\varphi: K \rightarrow L$, we find a continuous surjective homomorphism of $K$ onto $\mathbb{L}_{p}^{\omega_{1}}$.

(2) follows from Lemma 4.5.

It follows from [11, Theorem 35.2] that the quotient $L=K / N$ with the properties (a)-(c) from Proposition 4.6 is uniquely determined up to topological isomorphisms.

Claim 4.7. Let $K$ be an abelian pro-p-group and let $N$ be a closed subgroup of $K$ isomorphic to $\mathbb{Z}_{p}^{\sigma}$, where $\sigma$ is a cardinal $>\omega$. Then there exists a continuous surjective homomorphism of $K$ onto $\mathbb{L}_{p}^{\sigma}$.

Proof. Let $N=\prod_{n=1}^{\infty} N_{n}$, where each $N_{n} \cong \mathbb{Z}_{p}^{\sigma}$, and $M=\prod_{n=1}^{\infty} p^{n} N_{n}$. Then $N / M \cong \prod_{n=1}^{\infty} \mathbb{Z}\left(p^{n}\right)^{\sigma}=\mathbb{L}_{p}^{\sigma}$. Let $K_{0}=K / M$. Then $K_{0}\left[p^{n}\right] \supseteq(N / M)\left[p^{n}\right]$ and the last group contains a subgroup isomorphic to $\mathbb{Z}\left(p^{n}\right)^{\sigma}$ for every $n \in \mathbb{N}_{+}$. Hence

$$
w\left(p^{n-1} K_{0}\left[p^{n}\right]\right) \geq \sigma .
$$

By Proposition 4.6 there exists a closed subgroup $N_{0}$ of $K_{0}$ such that $N_{0} \cong \mathbb{Z}_{p}^{\sigma_{1}}$, $L_{0}=K_{0} / N_{0} \cong \prod_{n=1}^{\infty} \mathbb{Z}\left(p^{n}\right)^{\beta_{n}}$ for appropriate cardinals $\sigma_{1}, \beta_{n}$ and the canonical projection $\varphi: K_{0} \rightarrow L_{0}$ satisfies $\varphi\left(t\left(K_{0}\right)\right)=t\left(L_{0}\right)$. Since $K_{0}\left[p^{n}\right]$ is compact and trivially meets $N_{0}=\operatorname{ker} \varphi$, so $\varphi\left\lceil_{K_{0}\left[p^{n}\right]}: K_{0}\left[p^{n}\right] \rightarrow L_{0}\left[p^{n}\right]\right.$ is a topological isomorphism. Consequently $p^{n-1} L_{0}\left[p^{n}\right]$ is topologically isomorphic to $p^{n-1} K_{0}\left[p^{n}\right]$ and hence $w\left(p^{n-1} L_{0}\left[p^{n}\right]\right) \geq \sigma$ for every $n \in \mathbb{N}_{+}$by (1). Therefore $\sup _{n \geq m} \beta_{n} \geq \sigma$ for every $m \in \mathbb{N}_{+}$.

Let us prove that there exists a continuous surjective homomorphism $f: L_{0} \rightarrow$ $\mathbb{L}_{p}^{\sigma}$. Then combining it with $\varphi$ and with the canonical projection of $K$ onto $K_{0}=$ $K / M$ we are done. Note that infinitely many $\beta_{n}$ are infinite. So it is possible to suppose without loss of generality that all $\beta_{n}$ are infinite. If there are infinitely many $\beta_{n}$ such that $\beta_{n} \geq \sigma$, it is possible to find the required continuous surjective homomorphism $f: L_{0} \rightarrow \mathbb{L}_{p}^{\sigma}$. Otherwise there exists $n_{0} \in \mathbb{N}_{+}$such that $\omega \leq \beta_{n}<\sigma$ for every $n \geq n_{0}$, with $\sup _{n \geq n_{0}} \beta_{n}=\sigma$. Take an increasing subsequence $\left\{\beta_{n_{k}}\right\}_{k}$ of 
$\left\{\beta_{n}\right\}_{n}$ such that $\sup _{n_{k} \geq n_{0}} \beta_{n_{k}}=\sigma$. Observe that $\prod_{k=1}^{\infty} \mathbb{Z}\left(p^{n_{k}}\right)^{\beta_{n_{k}}}=\prod_{k=1}^{\infty} S_{k}^{\beta_{n_{k}}}$, where $S_{k}=\prod_{i=k}^{\infty} \mathbb{Z}\left(p^{n_{i}}\right)$ is a non-torsion metrizable compact abelian group. For every $k \in \mathbb{N}_{+}$there exists a continuous surjective homomorphism of $S_{k}$ onto $\mathbb{L}_{p}$. This gives rise to the desired continuous surjective homomorphism $f: L_{0} \rightarrow \mathbb{L}_{p}^{\sigma}$.

\section{Proof of the Main Theorem and Example 1.6}

The next lemma will be used in the proof of the Main Theorem 5.2. It settles the problem in the "local case", that is, when $K$ is an abelian pro- $p$-group.

Lemma 5.1. Let $K$ be an abelian pro-p-group. Then the following properties are equivalent:

(a) $K$ is non-singular;

(b) $K$ has the property $T D_{\omega}$;

(c) there exists a continuous surjective homomorphism of $K$ onto $\mathbb{L}_{p}^{\omega_{1}}$.

Proof. (a) $\Rightarrow$ (c) follows from Proposition 4.6 and Claim 4.7 and (c) $\Rightarrow$ (b) from Proposition 2.4 and Lemma 2.5.

(b) $\Rightarrow$ (a) The property $T D_{\omega}$ implies that $K$ has a proper totally dense pseudocompact subgroup. Now apply Theorem 1.3 and Lemma 3.3 to conclude that $K$ is non-singular.

Now it is possible to prove our Main Theorem. For technical convenience we add the equivalent conditions (c) and (e) that may also have independent interest.

Theorem 5.2. For a compact abelian group $K$ the following conditions are equivalent:

(a) $K$ has a proper totally dense pseudocompact subgroup;

(b) $K$ has no closed torsion $G_{\delta}$-subgroups;

(c) $K$ is non-singular;

(d) $K$ has the property $T D_{\omega}$;

(e) there exists a continuous surjective homomorphism of $K$ onto $S^{\omega_{1}}$ where $S$ is compact non-torsion.

Proof. (e) $\Rightarrow$ (d) by Proposition 2.4 and Lemma 2.5, (d) $\Rightarrow$ (a) is obvious, while $(\mathrm{a}) \Rightarrow(\mathrm{b})$ by Theorem 1.3 and $(\mathrm{b}) \Leftrightarrow(\mathrm{c})$ by Lemma 3.3 .

(c) $\Rightarrow(\mathrm{e})$ If $c(K)$ is not metrizable, then one can take $S=\mathbb{T}$ (see the proof of Theorem 1.2). Suppose that $w(c(K)) \leq \omega$. So there is a continuous surjective homomorphism of $K$ onto the non-singular totally disconnected group $K / c(K)$. Hence we can suppose without loss of generality that $K$ is totally disconnected and $K=\prod_{p \in \mathbb{P}} K_{p}$. If $K_{p}$ is non-singular for some $p \in \mathbb{P}$, apply Lemma 5.1. If $K_{p}$ is singular for every $p \in \mathbb{P}$, then $P_{s}=\mathbb{P}$ and $\mathbb{P} \backslash P_{m}$ has to be infinite by Lemma 4.4. Now Proposition 4.3 applies.

Remark 5.3. (1) As mentioned in [5], it is possible to prove that the following condition is equivalent to those of Theorem 5.2:

(f) $K$ has a totally dense subgroup $H$ that contains an $\omega$-bounded subgroup $M$ of $K$ such that $\bar{M}$ is a $G_{\delta}$-subgroup of $K$ and $\bar{M} \nsubseteq H$.

(2) For compact abelian groups this condition (f), as well as condition (a) of Theorem 5.2, is preserved under taking the inverse image by continuous surjective homomorphisms. 
Construction of Example 1.6. Consider the compact abelian group $K=\mathbb{Z}_{p}^{\omega_{1}}$. By LH we have $|K|=\mathfrak{c}$. Call a non-limit cardinal $\alpha=\lambda+n$, where $\lambda$ is a limit cardinal and $n \in \mathbb{N}_{+}$, odd if $n$ is odd and even if $n$ is even. Let $\mathcal{N}=\left\{N_{\lambda}\right.$ : $\lambda$ odd, $\lambda<\mathfrak{c}\}$ be the set of all closed subgroups of $K$ isomorphic to $\mathbb{Z}_{p}$. Let then $\mathcal{G}=\left\{O_{\nu}: \nu\right.$ even, $\left.\nu<\mathfrak{c}\right\}$ be the set of all cosets of $G_{\delta}$-subgroups of $K$.

We define $F_{0}=\{0\}$. For $0<\xi<\mathfrak{c}$ suppose that for every non-limit cardinal $\eta<\xi$, if $\eta$ is odd, there exists $x_{\eta} \in N_{\eta}$ such that $\overline{\left\langle x_{\eta}\right\rangle}=N_{\eta}$, and if $\eta$ is even, there exists $x_{\eta} \in O_{\eta}$ (if $\eta<\xi$ is a limit cardinal put $x_{\eta}=0$ ) and they have the property that $F_{\xi}=\left\langle x_{\eta}: \eta<\xi\right\rangle$ is free; observe that $\left|F_{\xi}\right|<\mathfrak{c}$ since $\xi<\mathfrak{c}$. If $\xi$ is odd, since $\left|N_{\xi}\right|=\mathfrak{c}$, we can choose $x_{\xi} \in N_{\xi}$ such that $\left\langle x_{\xi}\right\rangle \cap F_{\xi}=\{0\}$ and $\overline{\left\langle x_{\xi}\right\rangle}=N_{\xi}$. Indeed, we can choose $x_{\xi}^{*} \in N_{\xi}$ such that $\left\langle x_{\xi}^{*}\right\rangle \cap F_{\xi}=\{0\}$ but it can happen that $x_{\xi}^{*} \in p N_{\xi}$; in this case there exists $n \in \mathbb{N}_{+}$such that $x_{\xi}^{*} \notin p^{n} N_{\xi}$ and so we take a $x_{\xi} \in N_{\xi} \backslash p N_{\xi}$ with $x_{\xi}^{*}=p^{n-1} x_{\xi}$. If $\xi$ is even, since $\left|O_{\xi}\right|=\mathfrak{c}$, we can choose $x_{\xi} \in O_{\xi}$ such that $\left\langle x_{\xi}\right\rangle \cap F_{\xi}=\{0\}$. In both cases $F_{\xi+1}$ is free. Finally define $F_{\mathfrak{c}}=\left\langle x_{\xi}: 0<\xi<\mathfrak{c}\right\rangle$. Then $F_{\mathfrak{c}}$ is free and it is totally dense and $G_{\delta}$-dense (so pseudocompact) in $K$. Since it is free, $F_{\mathfrak{c}}$ cannot contain any non-trivial $\omega$-bounded subgroup and it is proper in $K$.

\section{ACKNOWLEDGMENTS}

It is a pleasure to thank the referee for her/his careful reading and helpful comments.

\section{REFERENCES}

1. J. Braconnier, Sur les groupes topologiques localement compacts (French), J. Math. Pures Appl. (9) 27 (1948), 1-85. MR0025473 (10:11c)

2. W. W. Comfort and L. C. Robertson, Cardinality constraints for pseudocompact and for totally dense subgroups of compact topological groups, Pacific J. Math. 119 (1985), 265-285. MR803119 (87i:22002)

3. W. W. Comfort and K. Ross, Pseudocompactness and uniform continuity in topological groups, Pacific J. Math. 16 (1966), 483-496. MR0207886 (34:7699)

4. W. W. Comfort and T. Soundararajan, Pseudocompact group topologies and totally dense subgroups, Pacific J. Math. 100 (1982), 61-84. MR661441 (83m:22008)

5. D. Dikranjan, Countably compact groups satisfying the open mapping theorem, Topology and its Applications 98 (1999), 81-129. MR1719996 (2000m:22005)

6. D. Dikranjan, A. Giordano Bruno and C. Milan, Weakly metrizable pseudocompact groups, Appl. General Topology 7 no. 1 (2006), 1-39. MR2284933

7. D. Dikranjan and Iv. Prodanov, Totally minimal groups, Ann. Univ. Sofia, Fac. Math. Méc. 69 (1974/75), 5-11. MR562518 (81c:22003)

8. D. Dikranjan, Iv. Prodanov and L. Stoyanov, Topological Groups: Characters, Dualities and Minimal Group Topologies, Pure and Applied Mathematics, Vol. 130, Marcel Dekker Inc., New York-Basel (1989). MR1015288 (91e:22001)

9. D. Dikranjan and D. Shakhmatov, Compact-like totally dense subgroups of compact groups, Proc. Amer. Math. Soc. 114 no. 4 (1992), 1119-1129. MR1081694 (92g:22009)

10. R. Engelking, General Topology, Heldermann Verlag, Berlin (1989). MR1039321 (91c:54001)

11. L. Fuchs, Infinite abelian groups vol. I, Academic Press, New York and London (1973). MR0349869 (50:2362)

12. A. Giordano Bruno, Dense minimal pseudocompact subgroups of compact abelian groups, Topology and Its Applications (to appear).

13. G. Grant, Topological groups which satisfy an open mapping theorem, Pacific J. Math. 68 (1977), 411-423. MR0466395 (57:6275)

14. E. Hewitt and K. Ross, Abstract harmonic analysis I, Springer-Verlag, Berlin-Heidelberg-New York (1963). MR0156915 (28:158) 
15. T. Soundararajan, Totally dense subgroups of topological groups, General Topology and Its Relations to Modern Analysis and Algebra III, Proc (1968) Kanpur Topological Conference, pp. 299-300, Academia Press, Prague (1971).

Dipartimento di Matematica e Informatica, Universitì di Udine, Via delle Scienze 206, 33100 Udine, ITALY

E-mail address: dikranja@dimi.uniud.it

Dipartimento di Matematica e Informatica, Università di Udine, Via delle Scienze 206, 33100 Udine, ItALY

E-mail address: giordano@dimi.uniud.it 